\title{
Prevention of gallstones by Lidan Granule: Insight into underlying mechanisms using a guinea pig model
}

\author{
XIAO WU ${ }^{1 *}$, XIAOQIANG LIANG ${ }^{2 *}$, YIJIE DU $^{1}$, YAN ZHANG $^{3}$, MENG YANG $^{3}$, WEIYI GONG $^{1}$, \\ BAOJUN LIU ${ }^{1}$, JINGCHENG DONG ${ }^{1}$, NINGXIA ZHANG ${ }^{4}$ and HONGYING ZHANG ${ }^{1,4}$ \\ ${ }^{1}$ Department of Integrative Medicine, Huashan Hospital, Fudan University, Shanghai 200040; \\ ${ }^{2}$ Longhua Hospital, Shanghai University of Traditional Chinese Medicine, Shanghai 200032; \\ ${ }^{3}$ General Surgery, Dong'e People's Hospital, Liaocheng, Shandong 252200; ${ }^{4}$ Putuo Hospital Affiliated to \\ Shanghai University of Traditional Chinese Medicine, Shanghai 200062, P.R. China
}

Received March 11, 2016; Accepted April 28, 2016

DOI: $10.3892 /$ br.2016.672

\begin{abstract}
The aim of the study was to examine the mechanism of action of Lidan Granule (LDG) for the prevention of gallstones using a guinea pig model. One hundred guinea pigs were divided into five groups randomly: control (standard diet and saline), model [lithogenic diet (LD) and saline], LDG-H (LD and $2 \mathrm{~g} / \mathrm{kg}$ of LDG), LDG-L (LD and $1 \mathrm{~g} / \mathrm{kg}$ of LDG), and ursodeoxycholic acid (UDCA) (LD and UDCA) as the positive control. At 6 weeks, the rate of gallstone formation and weight of the adrenal gland were recorded and serum levels of inflammatory cytokines were measured. Levels of corticotrophin-releasing hormone $(\mathrm{CRH})$ in the hypothalamus, adrenocorticotropic hormone (ACTH) in the hypophysis, and serum cortisol were determined. Bile components were tested with colorimetry. At 6 weeks, the rate of gallstone formation was significantly decreased in the LDG-H (14.29\%) and LDG-L (21.43\%) groups compared to the model group $(81.25 \%$; $\mathrm{P}<0.01)$. LDG treatment decreased the serum levels of interleukin (IL)-1, IL-6, and tumor necrosis factor- $\alpha(\mathrm{P}<0.01)$. LDG decreased bile cholesterol and increased bile acid and phospholipid levels in the bile $(\mathrm{P}<0.01)$. LDG treatment recovered the function of the hypothalamic-pituitary-adrenal (HPA) axis by increasing the expression of CRH $(\mathrm{P}<0.01)$ and ACTH $(\mathrm{P}<0.05)$. LDG made the bile less lithogenic, improved the function of the HPA axis, and regulated the expression of inflammatory cytokines for the prevention of cholelithiasis.
\end{abstract}

Correspondence to: Dr Hongying Zhang, Putuo Hospital Affiliated to Shanghai University of Traditional Chinese Medicine, 164 Lan Xi Road, Shanghai 200062, P.R. China

E-mail: zhangningxia1017@126.com

${ }^{*}$ Contributed equally

Key words: Lidan Granule, hypothalamic-pituitary-adrenal axis, pigment gallstones, inflammation

\section{Introduction}

Gallstone disease is one of the most common gastrointestinal disorders encountered in clinical practice $(1,2)$. Gallstones are often associated with the bile duct or intrahepatic stones, which have difficulty clearing the biliary system and are associated with a high rate of recurrence (3). Thus, prevention of the formation and recurrence of gallstones is necessary (4-7).

Gallstones are formed for many reasons. One cause is inflammation, which often changes the bile acid composition and accelerates stone formation $(8,9)$. Previous findings have shown that inflammatory cytokines such as lipopolysaccharide (LPS), tumor necrosis factor (TNF), and interleukin-1 (IL-1) can inhibit the production of bile acid through classic and bypass pathways (9-11). Inflammatory cytokines can disturb cholate transport in the hepatocytes and reduce the secretion of bile salts and phospholipids (9).

Apart from inflammation, an imbalance in bile components such as cholesterol, bile acids, and lecithin, also contributes to gallstone formation. As the concentration of cholesterol in bile increases, the bile begins to be saturated with cholesterol, and at a certain level, the cholesterol begins to crystallize. McNeilly et al (12) found that bile acids are involved in the regulation of glucocorticoid metabolism within the liver of female patients $(12,13)$. The increased level of bile acids during cholestasis may induce the downregulation of the hypothalamic-pituitary-adrenal (HPA) axis. The HPA axis plays a role in the body's response to stress by mediating the secretion of the adrenocorticotropic hormone (ACTH). Clinical studies have shown that gallstone disease is associated with dysregulation of the HPA axis with abnormal secretion of serum corticosterone (14). Thus, homeostasis of the HPA axis is important for the prevention of pigment gallstones.

In traditional Chinese medicine, gallstone refers to 'rib pain' or 'liver bilges', and the treatment of gallstones is based on the principle of 'liver-dispersing and bile discharging,' i.e., stone clearance and restoration of liver function. Lidan Granule (LDG) is formulated on the basis of these principles and consists of 15 types of Chinese herbs. It has been used for many years in clinical practice to treat and prevent gallstones $(4,15,16)$. The aim of the present study was to investigate 
Table I. Components and efficacy of LDG.

\begin{tabular}{|c|c|c|c|}
\hline English & Latin & Species & Effect \\
\hline Oriental wormwood & Artemisia capillaris Thunb & Origanum L. & Clear away heat and promote diuresis \\
\hline Hawthorn fruit & Crataegus pinnatifida Bunge & Crataegus L. & Aid digestion, increase appetite \\
\hline Rice sprout & Setaria italica & Rice & Aid digestion, increase appetite \\
\hline Germinated barley & Hordeurn vulgare L. & Gramineae L. & Aid digestion, increase appetite \\
\hline Green orange peel & Citrus reticulata Blanco & Citrus L. & Relieve food retention \\
\hline Tangerine peel & Citrus reticulata Blanco & Citrus L. & Dispel moisture and eliminate phlegm \\
\hline Medicated leaven & Massa Medicata Fermentata & Powder & Digestion \\
\hline Cyperus tuber & Cyperus rotundus $\mathrm{L}$. & Cyperus Linn. & Relieve chest and abdominal pain \\
\hline Radish seed & Semen Raphani & R. sativus L. & Relieve abdominal distention \\
\hline Caulis perillae & Perilla frutescens (L.) Britton. & P. frutescens L. Britt. & Relieve nausea \\
\hline Turmeric root & Curcuma longa $\mathrm{L}$. & Curcuma L. & Relieve abdominal pain \\
\hline Rhubarb & Rheum palmatum $\mathrm{L}$. & Rhubarb L. & Clear heat-fire \\
\hline Pinellia tube & Pinellia ternata (Thunb.) Breit. & Pinellia Ten. & Antiemetic effects \\
\hline Chinese honeylocust fruit & Gleditsia sinensis Lam. & Gleditsia Linn & Aid digestion; increase appetite \\
\hline
\end{tabular}

LDG, Lidan Granule.

Table II. Components of lithogenic diet food.

\begin{tabular}{|c|c|c|c|c|c|}
\hline Name & Dosage $(\mathrm{g} / \mathrm{kg})$ & Name & Dosage $(\mathrm{g} / \mathrm{kg})$ & Name & Dosage $(\mathrm{g} / \mathrm{kg})$ \\
\hline Corn flour & 136.3 & Alfalfa meal & 416.5 & Cellulose & 20 \\
\hline Salt & 10 & Whole wheat & 90.9 & Cholesterol & 1 \\
\hline Flour & 90.7 & Yeast powder & 10 & Vitamin C & 0.05 \\
\hline Soy bean flour & 90.9 & Lard oil & 20 & Cholic acid & 0.4 \\
\hline Fish meal & 63.6 & Sucrose & 20 & Casein & 20 \\
\hline
\end{tabular}

the role of LDG in the treatment and prevention of pigment gallstones and to explore the underlying mechanisms using a guinea pig model.

\section{Materials and methods}

Drugs and reagents. LDG was provided by the Department of the Integrative Medicine, Huashan Hospital, Fudan University (Shanghai, China), and the components and efficacy of LDG are described in Table I. Ursodeoxycholic acid (UDCA) was purchased from Sanwei Changjiang Biochemical Pharmaceutical Factory (Shanghai, China), and the enzymelinked immunosorbent assay (ELISA) kits were obtained from eBioscience, Inc. (San Diego, CA, USA). The lithogenic diet food (the components of which are listed in Table II) was purchased from Trophic Animal Feed High-tech Co., Ltd. (Jiangsu, China). LDG and UDCA were diluted in sterile saline. Animals in the LDG-H (2 g/kg/day, determined by the dosage used for humans clinically), LDG-L ( $1 \mathrm{~g} / \mathrm{kg} / \mathrm{day})$, and UDCA (50 mg/kg/day) groups were fed twice per day.

Animals and treatments. One hundred male guinea pigs (weighing 230-250 g) were obtained from the Shanghai SLAC Laboratory Animal Co., Ltd. (Shanghai, China). Animals were maintained in a temperature- $\left(20 \pm 2^{\circ} \mathrm{C}\right)$ and humidity- (50-60\%) controlled facility upon arrival on a 12-h light/dark cycle (lights on from 7:00 a.m. to 7:00 p.m.) and given access to food and water ad libitum. Animals were randomly divided into five groups. The control group mice were fed a chow diet, whereas mice in the other groups were fed a high cholesterol lithogenic diet $(17,18)$. The LDG-H group was fed with a lithogenic diet and LDG ( $2 \mathrm{~g} / \mathrm{kg} /$ day $)$, given orally, and the LDG-L group was fed the lithogenic diet and LDG (1 g/kg/day), given orally (Fig. 1). The UDCA group was fed UDCA (50 mg/kg/day), and the model group was fed with lithogenic diet and saline. Experiment animals were housed for a minimum of 7 days prior to the start of the experiment to adapt to the environment. Experiments were conducted in accordance with the guidelines of the Animal Care and Use Committee of Fudan University.

Serum, bile, and histological sample preparation. The animals were sacrificed by an overdose of sodium pentobarbital (50 mg/kg body weight, i.p.), and blood samples were obtained from the aorta abdominal and clotted for $2 \mathrm{~h}$ at room temperature before centrifugation $\left(1,006.2 \times \mathrm{g}\right.$ at $4^{\circ} \mathrm{C}$ for $10 \mathrm{~min})$. The supernatant was subsequently collected and stored at $-80^{\circ} \mathrm{C}$ for measurement of the levels of IL-6, IL-1, TNF- $\alpha$, and cortisol (CORT) in the peripheral blood. The guinea pigs were decapitated, and the brain tissues were 


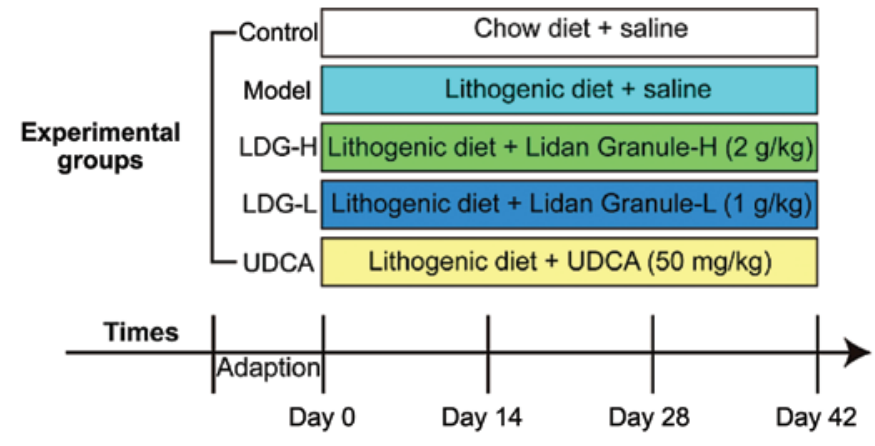

Figure 1. Schematic representation of the experiment.

removed and stored on ice. The bilateral hippocampus and hypophysis were rapidly and carefully removed using curved tweezers. Once snap frozen in liquid nitrogen, the brain tissue samples were stored at $-80^{\circ} \mathrm{C}$ until use. The adrenal glands of guinea pigs were also removed and weighed carefully.

Bile samples were obtained from dissected gall bladders, stored at $-20^{\circ} \mathrm{C}$, and diluted $1: 5$ with distilled water prior to analysis by ultra-performance liquid chromatography-mass spectrometry (UPLC-MS).

Physical state score (PSS). The physical states of the guinea pigs in the different groups were assessed as previously described (19). The physical states of guinea pigs were evaluated weekly until the end of the experiment. The coat state was recorded on a scale from 1 to 3 as follows: guinea pigs in a good state were scored as 3 (the fur was smooth with no tousling), animals in a bad state were scored as 1 (dirty fur on most of the body), and those with a coat state between 1 and 3 were scored as 2 . Each measurement was scored by another experimenter blinded to the treatment group.

Serum CORT, corticotrophin-releasing hormone (CRH), IL-6, and ACTH ELISA. Serum levels of CORT, IL-6, IL-1, and TNF- $\alpha$ were measured using a commercially available enzyme competitive ELISA test kit following the manufacturer's instructions (eBioscience, Inc.).

Statistical analysis. Data were presented as mean \pm standard error of the mean and analyzed with SPSS 17.0 software (SPSS, Inc., Chicago, IL, USA). The statistical significance of differences between the means of groups was determinant by one-way analysis of variance (ANOVA) followed by the least significant difference (LSD) for post-hoc comparisons. The rate of different groups were compared with the Chi-square test. $\mathrm{P}<0.05$ was considered to indicate a statistically significant difference.

\section{Results}

Effect on weight, PSS change, and the rate of gallstone formation. During 6 weeks of exposure to a lithogenic diet, guinea pigs in the model group showed a mild increase in weight, whereas those on low concentration $(1 \mathrm{~g} / \mathrm{kg})$ or high concentration $(2 \mathrm{~g} / \mathrm{kg})$ of LDG showed an obvious increase in weight after 2 weeks (Fig. 2A).

A

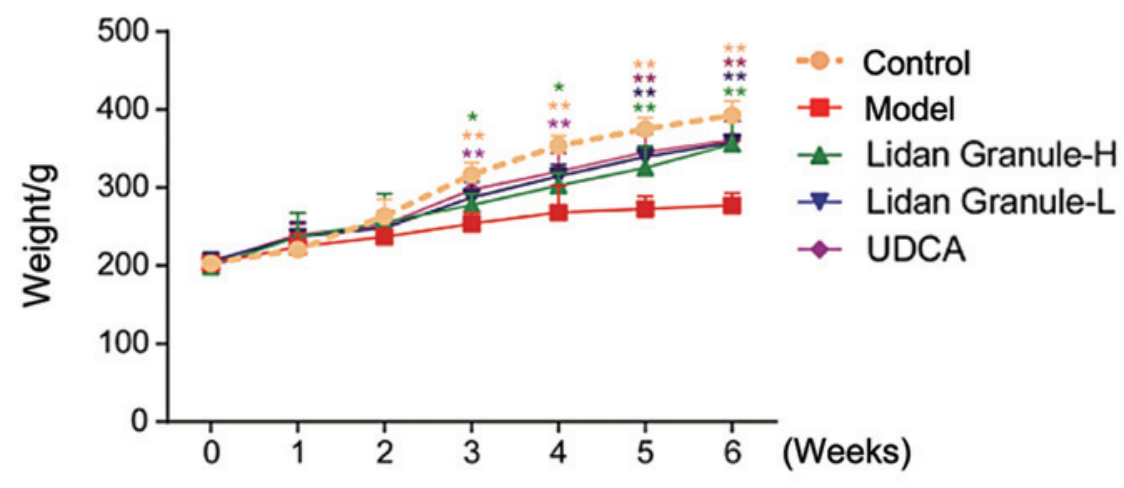

B

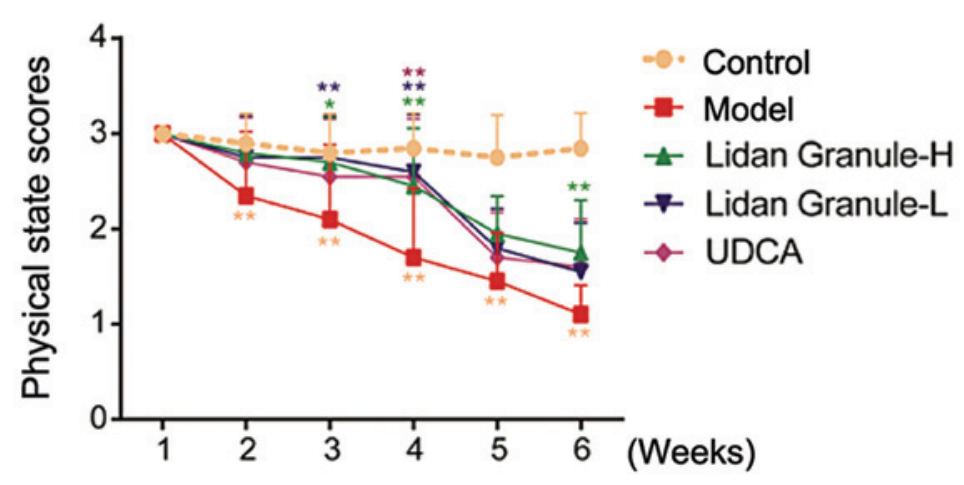

Figure 2. Trends in weight and PSS score of the guinea pigs. (A) Weight changes in the guinea pigs during the experiment. (B) The PSS score trend in the guinea pigs during the 6-week experiment. PSS, physical state score. 
Table III. Rates of gallstone formation in the different groups.

\begin{tabular}{lccc}
\hline Group & $\begin{array}{c}\text { No. of } \\
\text { animals }\end{array}$ & $\begin{array}{c}\text { No. of } \\
\text { gallstones }\end{array}$ & $\begin{array}{c}\text { Rate of gallstone } \\
\text { formation }(\%)\end{array}$ \\
\hline Control & 18 & 1 & $5.56^{\mathrm{b}}$ \\
Model & 16 & 13 & 81.25 \\
LDG-H & 14 & 2 & $14.29^{\mathrm{b}}$ \\
LDG-L & 14 & 3 & $21.43^{\mathrm{b}}$ \\
UDCA & 13 & 4 & $30.77^{\mathrm{a}}$ \\
\hline
\end{tabular}

Analysis by Chi-square test, compared with model group. ${ }^{\mathrm{a}} \mathrm{P}<0.05$, ${ }^{\mathrm{b}} \mathrm{P}<0.01$. LDG, Lidan Granule; UDCA, ursodeoxycholic acid.

Six weeks later, the differences in weights between the model and LDG groups were significant (both $\mathrm{P}<0.01$ ). The PSS score of the model group guinea pigs decreased during the 6-week experiment, and the PSS score was statistically different between the control and model groups $(\mathrm{P}<0.01)$ beyond the second week. The PSS scores for the treatment groups were reduced significantly during the fourth and fifth weeks (Fig. 2B).

Six weeks later, the rate of gallstone formation was $5.56 \%$ among 18 guinea pigs in the control group (Table III). For the model group, the stone formation rate was $81.25 \%$, which was significantly higher than that in the control group $(\mathrm{P}<0.01$; Table III). Four of 13 guinea pigs developed gallstones in the UDCA group $(\mathrm{P}<0.05)$, and the stone formation rates in the LDG-H and LDG-L groups were $14.29 \%$ $(\mathrm{P}<0.01)$ and $21.43 \%(\mathrm{P}<0.01)$, respectively. The rates were lower than the stone formation rate observed in the model group (Table III).

Effect on histopathological assessment of liver tissues. After 6 weeks of treatment, normal hepatocytes with blue-stained nuclei located in the center of the cell and arranged in cord-like patterns were evident in the control group (Fig. 3A and A1). In the pigment gallstone model group, hepatic steatosis in the form of fat globules in the hepatocytes with an eccentric nucleus and loose cytoplasm was observed. Many inflammatory cells were dispersed around the central vein, and small Mallory bodies were identified at higher magnification (Fig. 3B and B1). Compared to the control group, the LDG-H $(2 \mathrm{~g} / \mathrm{kg} / \mathrm{day}$; Fig. 3C and C1) and LDG-L (1 g/kg/day; Fig. 3D and D1) groups showed fewer inflammatory cells, and no obvious ballooning degeneration. Similar findings were observed in the UDCA group (Fig. 3E and E1), except for the presence of ballooning degeneration in a few areas around the central vein.

Effect of LDG on HPA axis activation. As shown in Fig. 4, 6 weeks of the lithogenic diet increased the CORT concentration in serum $(\mathrm{P}<0.01)$ compared to that in the control group and decreased the level of $\mathrm{CRH}$ in the hypothalamus $(\mathrm{P}<0.01)$ and expression of $\mathrm{ACTH}$ in the hypophysis $(\mathrm{P}<0.01)$. The lithogenic diet also had some effect on the weight of the adrenal gland $(\mathrm{P}<0.01)$. Six weeks of treatment with LDG ( 2 and $1 \mathrm{~g} / \mathrm{kg} / \mathrm{day}$ ) significantly improved the disturbance in the HPA axis caused by the lithogenic diet. Similarly, UDCA had a positive effect on the level of CORT in the peripheral blood $(\mathrm{P}<0.01)$ and the weight of the adrenal gland $(\mathrm{P}<0.01)$.

Effect of $L D G$ on $I L-6, I L-1$, and TNF- $\alpha$ levels in peripheral blood. The levels of IL-6, IL-1, and TNF- $\alpha$ in the peripheral blood were increased after 6 weeks on a lithogenic diet. Following treatment with high- or low-dose LDG, decreases in the serum concentration of IL-6 (Fig. 5A; P<0.01), IL-1 (Fig. 5B; $\mathrm{P}<0.01$ ), and TNF- $\alpha$ (Fig. 5C; $\mathrm{P}<0.01$ ) were observed.

Effect of LDG on the bile components. As expected, the lithogenic diet increased the concentration of cholesterol, direct bilirubin (DBIL), and indirect bilirubin (IBIL) in the bile and reduced the bile concentrations of bile acids and phospholipids (Fig. 6A and D). However, LDG significantly reduced the cholesterol, IBIL, and DBIL concentrations in the bile (Fig. 6B; $\mathrm{P}<0.01)$. At the same time, LDG increased the acid concentration in the bile (Fig. 6A; $\mathrm{P}<0.01$ ) after 6 weeks of treatment.

\section{Discussion}

The results of the present study suggest that a lithogenic diet can induce the formation of gallstones and disturb the HPA

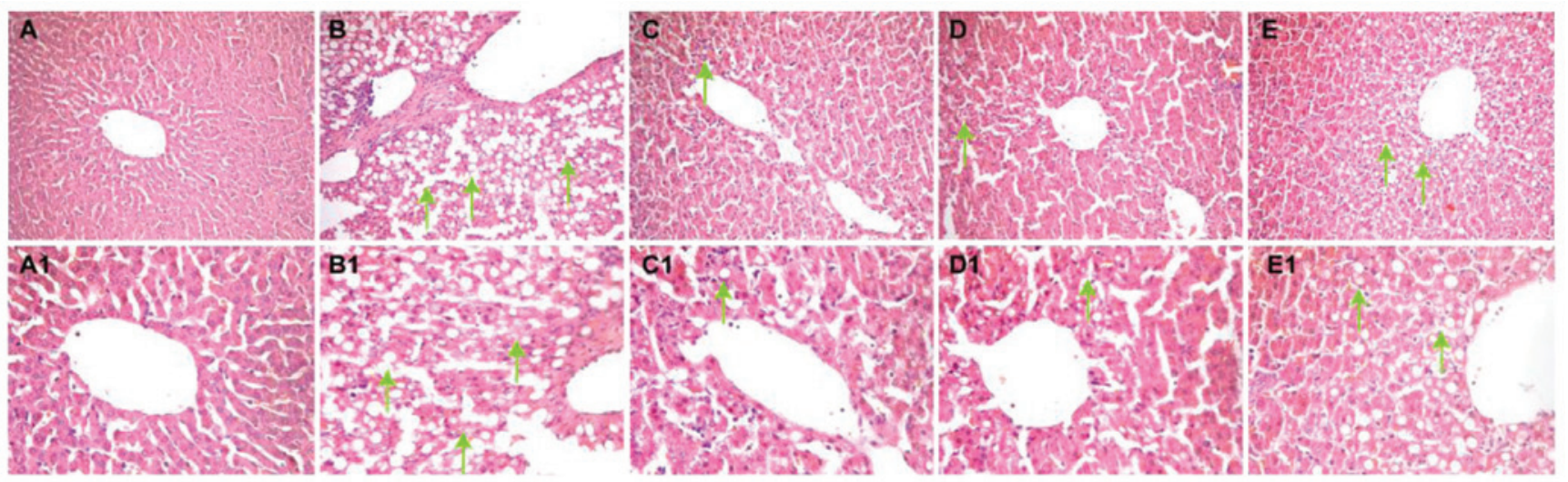

Figure 3. Histopathological findings in the liver of different groups. (A and A1) Sections of normal liver showing normal hepatocytes arranged in a cord-like pattern. (B and B1) Histological sections showing enlarged fatty hepatocytes with loose cytoplasm (green arrows) and eccentric nucleus (C and C1). (D and D1) Sections of liver after 6 weeks of exposure to LDG. (E and E1) Less loose cytoplasm and some ballooning degeneration in hepatocytes of the UDCA group (green arrows). LDG, Lidan Granule; UDCA, ursodeoxycholic acid. 

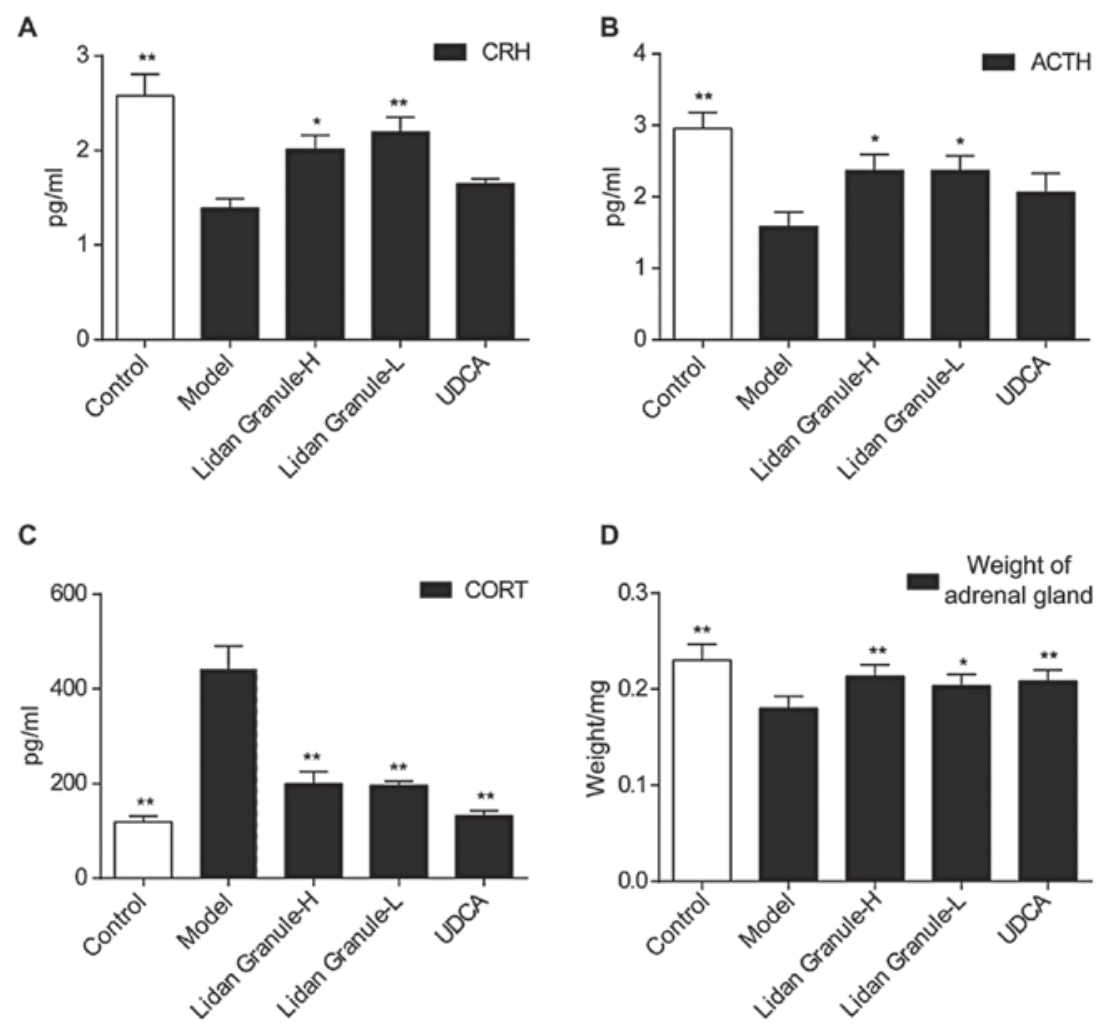

Figure 4. LDG administration partially improves the lithogenic diet-induced disturbance in the HPA axis. (A) LDG increased the expression of CRH and (B) ACTH but (C) reduced the production of CORT. (D) The effect of LDG on the weight of the adrenal gland. Data are the mean \pm SEM. ${ }^{*} \mathrm{P}<0.05$, ${ }^{* *} \mathrm{P}<0.01$ vs. model. LDG, Lidan Granule; HPA, hypothalamic-pituitary-adrenal; CRH, corticotrophin-releasing hormone; ACTH, adrenocorticotropic hormone; CORT, cortisol.
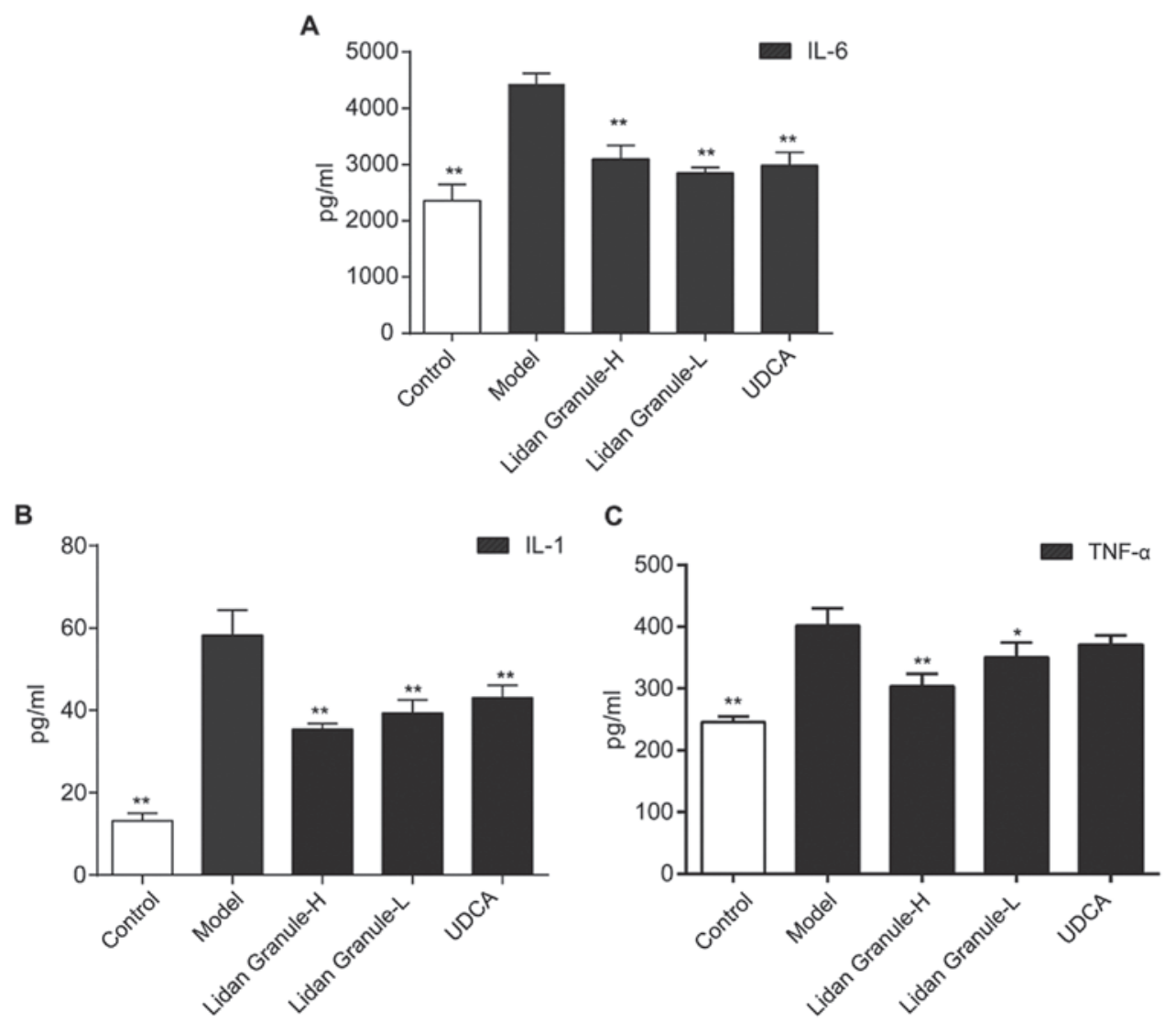

Figure 5. LDG improved the serum levels of IL-6, IL-1, and TNF- $\alpha$ in the guinea pigs. (A) Lithogenic diet induced an increase in IL-6 in the serum. LDG reduced the inflammatory response of the guinea pigs; (B) LDG improved the level of IL-1 in the peripheral blood. (C) LDG decreased the level of TNF- $\alpha$ in the serum. LDG, Lidan Granule; IL, interleukin; TNF, tumor necrosis factor. 

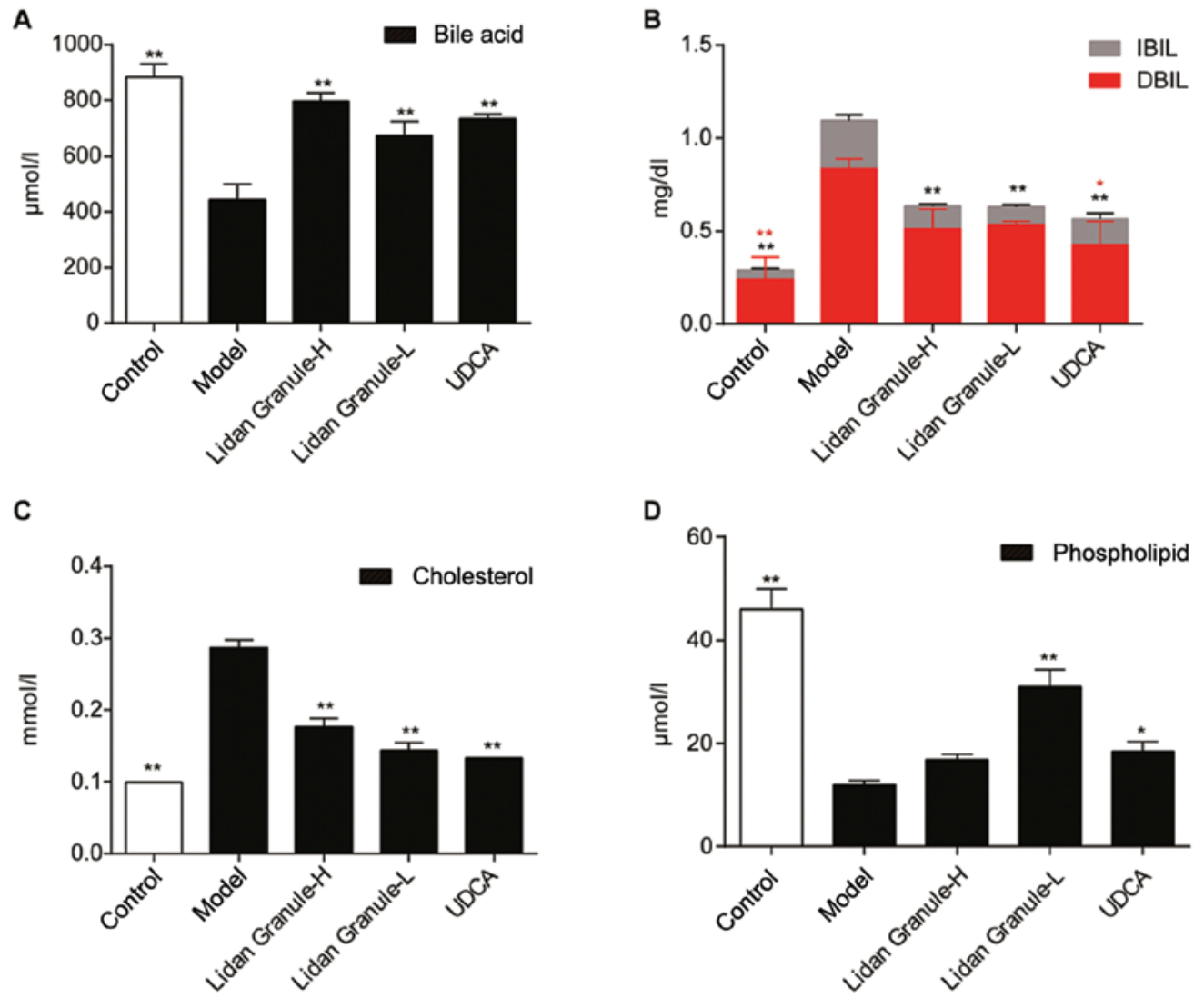

Figure 6. LDG ameliorated the effects of a lithogenic diet on the concentrations of bile acids, DBIL, IBIL, cholesterol, and phospholipids in the bile of guinea pigs. LDG, Lidan Granule; DBIL, direct bilirubin; IBIL, indirect bilirubin.

axis in guinea pigs, whereas LDG can ameliorate the effect of a lithogenic diet on the HPA axis and bile components.

Most gallstones are formed in the bile duct system, where they can block the bile duct and lead to cholestasis (20). During the disease process, cholestasis results in a decrease in liver function, and also, endocrine function (21). The diet is one of the most common pathogenic factors for gallstone formation. Thus, in our study, a lithogenic diet was given to guinea pigs for 6 weeks to induce the formation of pigment gallstones (22). After 6 weeks, serum IL-6, IL-1, and TNF- $\alpha$ levels in the model group were found to be elevated, while the histopathological examination of the livers showed hepatic steatosis and loose cytoplasm, with nuclei pushed to the edge of cells and inflammatory cell infiltration occurring. These findings confirm that the pigment gallstone model is suitable for studying the pathophysiology of pigment stone formation.

LDG has been used as a routine treatment for gallstones for many years, and clinical studies have demonstrated that LDG can promote biliary excretion and litholysis (15). LDG is composed of 15 types of Chinese herbs, namely, oriental wormwood, hawthorn fruit, rice sprout, germinated barley, green orange peel, tangerine peel, medicated leaven, cyperus tuber, radish seed, perilla stem, turmeric root, bitter orange, pinellia tube, and Chinese honey locust fruit, which is based on the Chinese medicinal principle of smoothening liver function, dredging the gallbladder, harmonizing the stomach, and strengthening the spleen (23).

The neuroendocrine system plays an important role in maintaining homeostasis (14). A clinical study has shown that gallstone formation in patients is always followed by endocrine disturbance, and changes in the corticosterone levels may indicate the presence of gallstone disease (24). In our study, after 6 weeks on a lithogenic diet, the CORT level in the model group was increased along with disruption of the HPA axis and a change in the weight of the adrenal gland. LDG treatment can ameliorate the secretion of proteins in the HPA axis, which means that LDG can regulate the neuroendocrine system during the process of gallstone formation.

IL-6 is one of the most important inflammatory biomarkers of cholelith disease $(9,25)$. In our study, serum IL-6 levels in the model group were elevated. Previous findings have shown that IL-6 in combination with other inflammatory cytokines or alone can influence the action of the HPA axis at different levels (26), which may explain the reason HPA axis dysfunction being considered in cholelith disease. LDG can improve the inflammatory state of guinea pigs by reducing the amounts of serum IL-6, IL-1, and TNF- $\alpha$, and improving the function of the neuroendocrine system at the same time.

Bile components are an important factor for the clinical diagnosis and differential diagnosis of gallstone disease $(27,28)$. In our study, the DBIL of the model group was higher than that of other groups, and it was in agreement with the clinical characteristics of silt-type cholesterol gallstones. After 6 weeks of LDG treatment, the DBIL was reduced significantly in the study group, which proves the dredging action of LDG.

In conclusion, the present study confirmed that cholelith disease is always accompanied by dysregulation of the HPA 
axis. LDG cannot only regulate the excretion of inflammatory factors but also improve the function of the neuroendocrine system, and improve the composition of the bile. LDG may influence the lithogenic character of bile via multiple pathways and has a definite role in the prevention and treatment of cholelithiasis.

\section{Acknowledgements}

The present study was funded by grants from the Development Project of Shanghai Peak Disciplines-Integrated Chinese and Western Medicine, the Doctoral Program of Higher Education of China (no. 20120071120076), the National Science Foundation for Distinguished Young Scholars of China (grant no. 81403148), and the National Science Foundation for Distinguished Young Scholars of China (grant no. 81501180).

\section{References}

1. Erichsen R, Frøslev T, Lash TL, Pedersen L and Sørensen HT: Long-term statin use and the risk of gallstone disease: a population-based case-control study. Am J Epidemiol 173: 162-170, 2011.

2. Stinton LM and Shaffer EA: Epidemiology of gallbladder disease: cholelithiasis and cancer. Gut Liver 6: 172-187, 2012

3. Markar SR, Karthikesalingam A, Thrumurthy S, Muirhead L, Kinross J and Paraskeva P: Single-incision laparoscopic surgery (SILS) vs. conventional multiport cholecystectomy: systematic review and meta-analysis. Surg Endosc 26: 1205-1213, 2012.

4. Zhang SB and Fang BJ: Effects of Yanggan Lidan Granule on rate of gallstone formation and content of plasma cholecystokinin in guinea pigs with induced cholesterol gallstones. Zhong Xi Yi Jie He Xue Bao 6: 405-408, 2008 (In Chinese).

5. Mann KS, Welfare E, Hughes D, et al: Significant service reconfiguration is required to definitively treat gallstone pancreatitis and prevent serious complications. Br J Surg 99: 164-165, 2012.

6. Smelt AH: Triglycerides and gallstone formation. Clin Chim Acta 411: 1625-1631, 2010 .

7. You SF, Zheng PY, Ji G, Wei HF, Zhao J and Zhu PT: Protective effects of yanggan lidan granules on carbon tetrachloride-induced liver damage in mice. Zhong Xi Yi Jie He Xue Bao 3: 470-472, 2005 (In Chinese).

8. Wang SN, Yeh YT, Wang ST, Chuang SC, Wang CL, Yu ML and Lee KT: Visfatin - a proinflammatory adipokine-in gallstone disease. Am J Surg 199: 459-465, 2010.

9. Maurer KJ, Carey MC and Fox JG: Roles of infection, inflammation, and the immune system in cholesterol gallstone formation. Gastroenterology 136: 425-440, 2009.

10. Kasprzak A, Szmyt M, Malkowski W, Przybyszewska W, Helak-Łapaj C, Seraszek-Jaros A, Surdacka A, Małkowska-Lanzafame A and Kaczmarek E: Analysis of immunohistochemical expression of proinflammatory cytokines (IL-1 $\alpha$, IL-6, and TNF- $\alpha$ ) in gallbladder mucosa: Comparative study in acute and chronic calculous cholecystitis. Folia Morphol (Warsz) 74: 65-72, 2015.

11. Chai J, He Y, Cai SY, Jiang Z, Wang H, Li Q, Chen L, Peng Z, He X, $\mathrm{Wu} \mathrm{X}$, et al: Elevated hepatic multidrug resistance-associated protein 3/ATP-binding cassette subfamily $\mathrm{C} 3$ expression in human obstructive cholestasis is mediated through tumor necrosis factor alpha and c-Jun NH2-terminal kinase/stress-activated protein kinase-signaling pathway. Hepatology 55: 1485-1494, 2012.
12. McNeilly AD, Macfarlane DP, O'Flaherty E, Livingstone DE, Mitić T, McConnell KM, McKenzie SM, Davies E, Reynolds RM, Thiesson HC, et al: Bile acids modulate glucocorticoid metabolism and the hypothalamic-pituitary-adrenal axis in obstructive jaundice. J Hepatol 52: 705-711, 2010.

13. Soliman HM, Abd El-Haleem MR and El Tarhouny SA; Histomorphometrical and Electron Microscopic Study of Adrenocorticocytes Following Surgically Induced Extrahepatic Biliary Obstruction in Adult Female Albino Rats: Histomorphometrical and electron microscopic study of adrenocorticocytes following surgically induced extrahepatic biliary obstruction in adult female albino rats. Folia Biol (Praha) 61: 14-25, 2015.

14. Rose AJ, Berriel Díaz M, Reimann A, Klement J, Walcher T, Krones-Herzig A, Strobel O, Werner J, Peters A, Kleyman A, et al: Molecular control of systemic bile acid homeostasis by the liver glucocorticoid receptor. Cell Metab 14: 123-130, 2011.

15. Xiang Y, Chen JH, Cai D and Ma BJ: Effect of Lidan Granule on bile lithogenesis in patients with choledocholithiasis combined with cholecystolithiasis. Chin J Integr Med 14: 142-144, 2008.

16. Zuo YT, Gao WY, Jia W, Duan HQ and Xiao PG: Prevention and treatment of cholelithiasis by traditional Chinese medicine. Zhongguo Zhong Yao Za Zhi 29: 831-833, 910, 2004 (In Chinese).

17. Wang HH, Portincasa P, Mendez-Sanchez N, Uribe M and Wang DQ: Effect of ezetimibe on the prevention and dissolution of cholesterol gallstones. Gastroenterology 134: 2101-2110, 2008.

18. Song XY, Xu S, Hu JF, Tang J, Chu SF, Liu H, Han N, Li JW, Zhang DM, Li YT, et al: Piperine prevents cholesterol gallstones formation in mice. Eur J Pharmacol 751: 112-117, 2015.

19. Alonso R, Griebel G, Pavone G, Stemmelin J, Le Fur G and Soubrié P: Blockade of CRF(1) or V(1b) receptors reverses stress-induced suppression of neurogenesis in a mouse model of depression. Mol Psychiatry 9: 278-286, 224, 2004.

20. Zhu L, Aili A, Zhang C, Saiding A and Abudureyimu K: Prevalence of and risk factors for gallstones in Uighur and Han Chinese. World J Gastroenterol 20: 14942-14949, 2014.

21. Wittenburg H: Hereditary liver disease: gallstones. Best Pract Res Clin Gastroenterol 24: 747-756, 2010.

22. Méndez-Sánchez N, Zamora-Valdés D, Chávez-Tapia NC and Uribe M: Role of diet in cholesterol gallstone formation. Clin Chim Acta 376: 1-8, 2007.

23. Fang BJ, Zhou S, Pei XJ, Huang JY, Chen BJ, Geng Y and Yang LK: Effects of Yanggan Lidan Granule on insulin resistance in guinea pigs with induced cholesterol gallstones. Zhong Xi Yi Jie He Xue Bao 7: 1159-1163, 2009 (In Chinese).

24. No authors listed: Current bibliographies of neuropeptides prepared by the University of Sheffield Biomedical Information Service. Neuropeptides 29: B37-B49, 1995.

25. Yun JW, Kim JS, Choi WS, et al: Inhibition of sphingolipid pathway affects the formation of cholesterol gallstone by the modulation of Il-6/Stat 3 pathway in mice model. J Gastroenterol Hepatol 27: 85-86, 2012.

26. Rosenblat JD, Cha DS, Mansur RB and McIntyre RS: Inflamed moods: a review of the interactions between inflammation and mood disorders. Prog Neuropsychopharmacol Biol Psychiatry 53: 23-34, 2014.

27. Peng Y, Yang Y, Liu Y, Nie Y, Xu P, Xia B, Tian F and Sun Q: Cholesterol gallstones and bile host diverse bacterial communities with potential to promote the formation of gallstones. Microb Pathog 83-84: 57-63, 2015.

28. Halilbasic E, Claudel T and Trauner M: Bile acid transporters and regulatory nuclear receptors in the liver and beyond. J Hepatol 58: 155-168, 2013. 